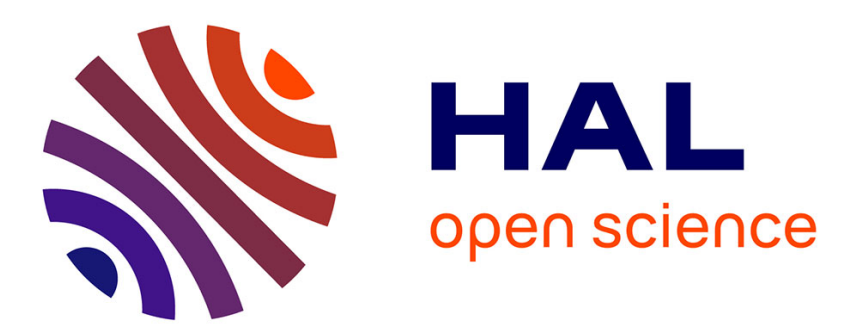

\title{
Pressure and shear stress caused by raindrop impact at the soil surface: Scaling laws depending on the water depth
}

\author{
Amina Nouhou Bako, Frédéric Darboux, Francois James, Christophe \\ Josserand, Carine Lucas
}

\section{To cite this version:}

Amina Nouhou Bako, Frédéric Darboux, Francois James, Christophe Josserand, Carine Lucas. Pressure and shear stress caused by raindrop impact at the soil surface: Scaling laws depending on the water depth. Earth Surface Processes and Landforms, 2016, 41 (9), pp.1199-1210. 10.1002/esp.3894 . hal-01281209v2

\section{HAL Id: hal-01281209 \\ https://hal.science/hal-01281209v2}

Submitted on 13 Feb 2017

HAL is a multi-disciplinary open access archive for the deposit and dissemination of scientific research documents, whether they are published or not. The documents may come from teaching and research institutions in France or abroad, or from public or private research centers.
L'archive ouverte pluridisciplinaire HAL, est destinée au dépôt et à la diffusion de documents scientifiques de niveau recherche, publiés ou non, émanant des établissements d'enseignement et de recherche français ou étrangers, des laboratoires publics ou privés. 


\title{
Pressure and shear stress caused by raindrop impact at the soil surface: Scaling laws depending on the water depth
}

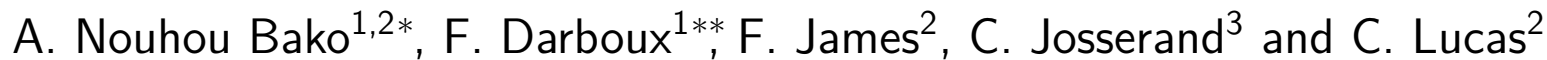 \\ ${ }^{1}$ Inra, UR0272, UR Science du sol, Centre de recherche Val de Loire, \\ CS 40001, F-45075 Orléans Cedex 2, France. \\ ${ }^{2}$ MAPMO, UMR CNRS 7349, Fédération Denis Poisson, FR CNRS 2964, \\ Université d'Orléans, F-45067 Orléans cedex 02, France. \\ 3 Sorbonne Universités, CNRS \& UPMC Univ Paris 06, UMR 7190, \\ Institut Jean Le Rond d'Alembert, F-75005, Paris, France.
}

\begin{abstract}
Raindrop impact is an important process in soil erosion. Through its pressure and shear stress, raindrop impact causes a significant detachment of the soil material, making this material available for transport by sheet flow. Thanks to the accurate Navier-Stokes equations solver Gerris, we simulate the impact of a single raindrop of diameter $D$, at terminal velocity, on water layers of different thickness $h: \frac{D}{10}, \frac{D}{5}, \frac{D}{3}, \frac{D}{2}, D, 2 D$, in order to study pressures and shear stresses involved in raindrop erosion. These complex numerical simulations help to understand precisely the dynamics of the raindrop impact, quantifying in particular the pressure and the shear stress fields. A detailed analysis of these fields is performed and self-similar structures are identified for the pressure and the shear stress on the soil surface. The evolution of these self-similar structures are investigated as the aspect ratio $h / D$ varies. We find that the pressure and the shear stress have a specific dependence on the ratio between the drop diameter and the water layer thickness and that the scaling laws recently proposed in fluid mechanics are also applicable to raindrops, paving the road to obtain effective models of soil erosion by raindrops. In particular, we obtain a scaling law formula for the dependance of the maximum shear stress on the soil on the water depth, quantity that is crucial for quantifying erosion materials.
\end{abstract}

Keywords: Raindrop, Navier-Stokes equations, pressure, shear stress

\footnotetext{
${ }^{*}$ Corresponding author: amina.nouhou-bako@orleans.inra.fr

** Presently at: Inra, Laboratoire Sols et Environnement, UMR 1120, Vandoeuvre-lès-Nancy, F-54518, France
} 


\section{Introduction}

Raindrop impact is a major driver of soil erosion and is acting through a wide range of processes (Terry, 1998; Planchon and Mouche, 2010): the raindrop impacts break down aggregates, leading to soil detachment and crust formation (Bresson and Moran, 2003). They also cause splashes, i.e. the transport of soil material in the air over distances of a few decimeters (Leguédois et al., 2005). Also, raindrop impacts are essential in shallow overland flow (i.e. sheet flow) for the detachment of particles. Indeed, sheet flow by itself does not have the ability to detach particles because of its limited velocity and thus weak shear stress (Kinnell, 1991). The impacts of the raindrops can detach the material that is then transported by the sheet flow.

Drop impact effects can differ strongly depending on whether the soil is dry or wet because both the shear strength of the soil and the shear stress caused by the drops depend on the soil humidity. Rapidly, for raindrops, the soil can be considered wetted so that we will focus here on the impact on a thin liquid film. The presence of a thin water layer at the soil surface modifies the effect of raindrop impacts (Kinnell, 1991). The consequences of drop impacts depend primarily on drop properties. However, the drops of concern for soil erosion have a narrow range of features: raindrops are considered at terminal velocity, leading to a clear relationship between diameter and velocity (Atlas et al., 1973). This contrasts with other usual applications in fluid mechanics (e.g. ink-jet printing where the ink drop impacts the paper or the coating of a surface by multiple drop impacts) where drops vary in viscosity, density, surface tension, velocity and diameter (Marengo et al., 2011).

Raindrop-driven erosion depends also on soil properties such as soil resistance to shear stress (Sharma et al., 1991; Mouzai and Bouhadef, 2011), hydrophobicity (Ahn et al., 2013) and roughness (Erpul et al., 2004). While raindrop impacts cause splashes, the quantity of eroded material is controlled mostly by the shear created by the impact, which is not strongly affected by the splash itself (Josserand and Zaleski, 2003). Indeed, it has been argued that the erosion, in term of bedload transport rate, is controlled by the shear stress affected at the soil boundary, usually measured through the dimensionless Shields number (Parker, 1990, Charru et al., 2004, Houssais and Lajeunesse, 2012). Although these results have been deduced precisely for gravel river beds made of non-cohesive granular materials with a narrow granulometric distribution, it is believed that this bed shear stress and to a smaller extent the bed pressure, are the main ingredients of most of erosion processes. Therefore, if the transport of eroded materials can be influenced by the splashing itself, which is always present for raindrop impact, the bedload transport rate is primarily due to the shear stress created by the impact. 
The influence of the water layer on the erosion process has also drawn attention: at first, one could argue that the erosion is limited by the shielding of the soil surface by the water layer. Raindrop energy is absorbed by the water layer, which lowers the pressure and shear stress exerted on the soil. It has been in fact documented that a water layer can maximize sheetflow erosion rate in comparison to a drained surface and that such erosion depends mostly on the ratio between the water depth and the raindrop diameter (Singer et al., 1981). In fact, there is a critical depth $h_{c}$ at which the splash transport rate is maximum: beyond $h_{c}$ the transport rate decreases strongly. However, different values for $h_{c}$ have been proposed in the literature as shown in Dunne et al. (2010): for instance it can vary from $h_{c}=D($ Palmer, 1963,1965$)$ to $h_{c}=0.2 D$ in Torri and Sfalanga (1986), and even with $0.14 D \leq h_{c} \leq 0.2 D$ according to Mutchler and Young (1975). Finally, Ghadiri and Payne (1986) showed a reduction of soil splash once a water layer covers the soil surface while Moss and Green (1983) and Kinnell (1991) found that the outflow rate of raindrop-induced flow transport reaches its maximum value when the flow depth equals two to three drop diameters. For three drop diameters (and above), detachment by raindrops becomes quite limited but drop energy still allows for particle suspension, leading to a significant transportation rate (Ferreira and Singer, 1985).

Raindrop interaction with the soil surface has been investigated using numerical simulations from the 1970 (Wang and Wenzel, Jr, 1970). They allow for the computation of pressure and shear stress fields at the soil surface (Huang et al., 1982: Ferreira et al., 1985; Hartley and Alonso, 1991; Hartley and Julien, 1992). All these simulations considered a rigid soil surface, hence not accounting for the elasticity of the soil or its granular nature. According to Ghadiri and Payne (1986), the soil behaves like a solid during the short time of the impact, justifying the simplification. These simulations have enabled the determination of the critical variables. For example, the maximum shear stress was found to depend mostly on the Reynolds number and of the water layer thickness-drop diameter ratio (Hartley and Alonso, 1991; Hartley and Julien, 1992). However, due to limitations in computer and algorithm performance, simulations were carried out with critical parameters (such as the Reynolds number) well out of the natural range, moderating confidence in the results.

The present paper takes advantage of the recent developments of detailed and direct simulations in fluid mechanics to study the impact of single raindrops on a soil surface with a water layer (see the reviews on drop impacts in the fluid mechanics literature: Rein (1993); Yarin (2006); Marengo et al. (2011); Josserand and Thoroddsen (2016)). The pressure field inside the water layer, the pressure field at the soil surface and the shear stress at the soil surface are analyzed for raindrop of diameter $2 \mathrm{~mm}$ and terminal velocity $6.5 \mathrm{~m} \cdot \mathrm{s}^{-1}$, varying the thickness of the water layer. Short time scales are considered, i.e. develop- 


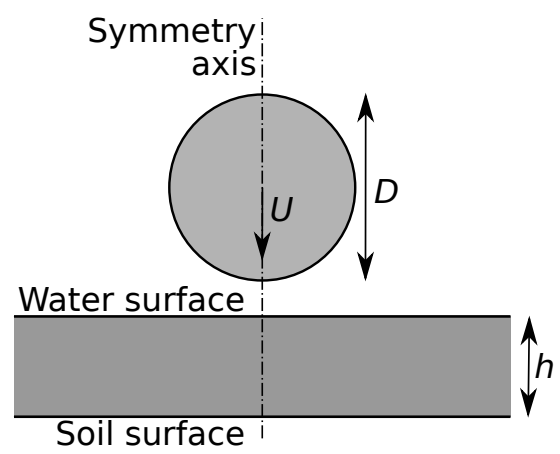

Figure 1: Schematic configuration of a raindrop (diameter $D$ and terminal velocity $U$ ) impacting a water layer of depth $h$.

ment of stresses before particle splash initiation. A self-similar approach valid for a thin liquid layer is used to analyze the results, showing that scaling laws recently proposed in fluid mechanics apply to natural raindrops too. It confirms that the ratio between the water depth and the raindrop diameter is critical to understand the effect of raindrop impact.

\section{Materials and Methods}

\section{Problem configuration}

We consider the normal impact of a liquid drop of diameter $D$ on a thin liquid film of thickness $h$ in the paradigm of rainfall (Figure 1 ). The liquid has a density and dynamic viscosity denoted $\rho_{l}$ and $\mu_{l}$. The density and viscosity of the surrounding gas are denoted $\rho_{g}$ and $\mu_{g}$. The drop impacts on the ground at velocity $\mathbf{U}=$ $-U_{0} \mathbf{e}_{z}$ which corresponds to the terminal velocity for a raindrop. We will assume here for the sake of simplicity that the raindrop has a spherical shape, even though it is known that raindrops can have a deformed shape, particularly for large diameters (Villermaux and Bossa, 2009). However, it is not expected to significantly impact the dynamics and the effect of the specific shape of the impacting drop is postponed to future work. The gravity is denoted $\mathbf{g}=-g \mathbf{e}_{z}$, and the liquid-gas surface tension $\gamma$.

Different dimensionless parameters can be constructed in this configuration. Two of them are commonly used in drop impact problems, since they characterize the balance between the inertia of the drop with the viscous and capillary forces respectively: firstly, the Reynolds number $R e$ which is the ratio between inertia and viscous forces:

$$
R e=\frac{\rho_{l} U_{0} R}{\mu_{l}}
$$


For raindrops, Re ranges from 6500 to 23000 (Hartley and Julien, 1992). Secondly the Weber number We which is defined as the ratio between inertia and capillary forces:

$$
W e=\frac{\rho_{l} U_{0}^{2} R}{\gamma}
$$

The Weber number ranges from 50 (for a raindrop diameter of $0.5 \mathrm{~mm}$ and a velocity of $2 \mathrm{~m} / \mathrm{s}$ ) to 12000 (for a raindrop diameter of $6 \mathrm{~mm}$ and a velocity of $9 \mathrm{~m} / \mathrm{s}$ ) for natural rainfall. The problem depends also on the aspect ratio of the problem geometry, i.e. the ratio between the drop diameter and the thickness of the liquid film:

$$
\frac{h}{D}
$$

Additional dimensionless numbers are present in this problem, but are of limited interest, either because they do not depend on the raindrop impact configuration, or because they characterize a physical mechanism that can be neglected here. It is the case of the Froude number

$$
F r=\frac{U_{0}^{2}}{g D}
$$

which quantifies the ratio between inertia and gravity forces, it can take values between 800 and 1400 for natural rainfall. Indeed, although gravity is crucial to accelerate the raindrop to its terminal velocity, the gravity itself plays quite a limited role in the impact dynamics and hence is usually not accounted for in the modeling and numerical simulation of drop impacts (Josserand and Zaleski, 2003). For instance, for a drop falling from a height $H$, the free fall velocity gives $U_{0}^{2} \sim 2 g H$ so that the Froude number is simply the ratio $2 H / D$. Since the terminal velocity of a raindrop is equivalent to a free fall height $H$ of several meters, the Froude number will always be very high in the present problem. Remark finally that while the Reynolds and Weber numbers are also high in the present problem, it does not mean that viscous and capillary effects can be neglected: the formation of a thin liquid layer means that viscous and capillary effects will be important in some region of the flows - something that is not valid for gravity, which can thus be safely neglected.

The two dimensionless numbers related to the liquid/gas properties, namely the density ratio $\rho_{g} / \rho_{l}$ and the viscosity ratio $\mu_{g} / \mu_{l}$, present a limited interest too: while the surrounding gas can sometime influence the splashing properties (Xu et al., 2005), in particular through the entrapment of an air bubble beneath the drop at the impact (Thoroddsen et al., 2003, 2005), this effect is negligible for the impact of a raindrop (Hartley and Alonso, 1991). Moreover, these two dimensionless numbers are only related to the gas and liquid characteristics and 
not to the impact conditions. Therefore they do not vary significantly with the raindrop radius and velocity.

Based on the water hammer pressure, that is the pressure created by the inertia of the drop hitting a solid surface, it was first suggested in soil erosion literature that the amplitude of the stress on the soil surface can be quite large $(2-6 \mathrm{MPa})$, with a limited duration of about 50 microseconds (Ghadiri and Payne, 1980), this duration increasing with the depth of the water layer (Ghadiri and Payne, 1986). However, such high water hammer pressures have not been observed experimentally (Ghadiri and Payne, 1986, Hartley and Julien, 1992; Josserand and Zaleski, 2003) and, following the argument of Ghadiri and Payne (1980), it can be shown that this pressure should arise only during a very short time of the order of $D / c$ (where $D$ is the drop diameter and $c$ the sound speed) leading to a typical time scale of the order of one microsecond as found by Ghadiri and Payne (1986). Then, the pressure decreases rapidly with time as shown in numerical simulations (Josserand and Zaleski, 2003). Moreover, as discussed by Nearing et al. (1986) and Nearing et al. (1987), compressible effects can be neglected since they will only influence the very early time of contact and a very small region of the impacted zone. This is in agreement with former theoretical and experimental studies on drop impacts where compressible effects were shown to appear only at much higher drop velocities, typically of the order of a fraction of the sound velocity in water (Lesser and Field, 1983). Therefore, as shown and as used in the recent studies, the liquid can be assumed incompressible during the impact (Rein, 1993; Yarin, 2006, Marengo et al., 2011; Josserand and Thoroddsen, 2016).

\section{The two-fluid Navier-Stokes equations}

Both the gas and the liquid obey the incompressible Navier-Stokes equations (with respective densities and viscosities) with jump conditions at the interface. This complete dynamics can be described within the one fluid formulation of the incompressible Navier-Stokes equation, that reads:

$$
\rho\left(\frac{\partial \mathbf{u}}{\partial t}+\mathbf{u} \nabla \mathbf{u}\right)=-\nabla p+\rho \mathbf{g}+\mu \nabla \cdot\left(\frac{\nabla \mathbf{u}+{ }^{t} \nabla \mathbf{u}}{2}\right)+\gamma \kappa \delta_{s} \mathbf{n}
$$

to which is added the equation of mass conservation, which for incompressible fluid yields

$$
\nabla \cdot \mathbf{u}=0,
$$

where $\mathbf{u}$ is the vector of fluid velocity, $\nabla$ is the usual differential operator, $p$ the pressure field, function of space $\mathbf{x}$ and time $t$. In these equations, the density $\rho(\mathbf{x}, t)$ and viscosity $\mu(\mathbf{x}, t)$ are discontinuous fields of space and time. $\rho(\mathbf{x}, t)$ 
$(\mu(\mathbf{x}, t))$ is $\rho_{l}$ or $\rho_{g}\left(\mu_{l}\right.$ or $\left.\mu_{g}\right)$ depending on whether we are in the liquid or gas phase. The term $\gamma \kappa \delta_{s} \mathbf{n}$ represents the surface tension force, proportional to the curvature $\kappa$ and localized on the interface (the Dirac term $\delta_{s}$ ) with normal is $\mathbf{n}$. The curvature is defined by the divergence of this vector:

$$
\kappa=\nabla \cdot \mathbf{n}
$$

An additional equation has to be considered for the motion of each phase (gas and liquid) leading eventually to the movement of the interface. Indeed, considering the characteristic function $\chi(\mathbf{x}, t)$ which is equal to one in the liquid phase and zero in the gas phase, the volume conservation of both phases implies that $\chi$ is solution of the advection equation:

$$
\frac{\partial \chi}{\partial t}+\mathbf{u} \cdot \nabla \chi=0
$$

Within this framework, both fluids satisfy the incompressible Navier-Stokes equation with the applicable density and viscosity.

From here on the soil surface is taken to be rigid. This simplification comes from the unavailability of a realistic deformation law for soils at the scale of a raindrop.

\section{Numerical Method and dimensionless version}

The Navier-Stokes equations (11,2,3) are solved by the open source Gerris flow solver (version 2013/12/06) (Popinet, 2007). Gerris uses the Volume of Fluid method on an adaptive grid (Popinet, 2003, 2009). The rotational symmetry of the problem around the vertical axis is used to perform 2D numerical simulations using cylindrical coordinates (called 3D-axisymmetric coordinates).

The discretization of the equations is made on a quadtree structure for square cells. The quadtree structure allows for a dynamic mesh refinement: when needed, a "parent" cell of the mesh is divided into four identical square "children" cells (which length is half the one of the parent cell), up to a maximum level $n$ of refinement. Similarly, a cell merging is performed whenever the precision of the computation is below a user-defined threshold. The refinement/merging criterion is based on a mix of high values of the density and velocity gradients. Hence, smaller cells are used at the gas/liquid interface and at locations showing large changes in velocity.

The interface between the gas and liquid phases is tracked using a color function $C$ which corresponds to the integral of the characteristic function in each grid cell. $C$ is taken as the fraction of liquid phase inside the cell. This allows for the interface to be reconstructed using the piecewise linear interface calculation 
(Li, 1995), leading to a conservative advective scheme for the advection of the interface (Brackbill et al., 1992; Lafaurie et al., 1994). For each phase, the viscosities $\left(\mu_{g}\right.$ or $\left.\mu_{l}\right)$ and the densities $\left(\rho_{g}\right.$ or $\left.\rho_{l}\right)$ are constant because the fluids are assumed incompressible. Hence, each cell crossed by the interface has a viscosity $\mu$ and a density $\rho$ determined by the relative volume fraction of each phase, following:

$$
\rho=C \rho_{l}+(1-C) \rho_{g} ; \text { and } \mu=C \mu_{l}+(1-C) \mu_{g} .
$$

Finally, the Navier-Stokes equations are solved in Gerris in a dimensionless form to lower numerical errors. The domain length has a size of one. The other lengths are rescaled by a factor $\lambda$ using a numerical diameter of the raindrop $D^{\prime}=0.3$ in Gerris (so $\lambda=D / D^{\prime}$ ), the velocities, densities, time and pressure by $U_{0}, \rho_{l}, \lambda / U_{0}$ and $\rho_{l} U_{0}^{2}$, respectively. Hence, the effective Navier-Stokes equation to solve reads:

$$
\rho^{\prime}\left(\frac{\partial \mathbf{u}^{\prime}}{\partial t^{\prime}}+\mathbf{u}^{\prime} \nabla^{\prime} \mathbf{u}^{\prime}\right)=-\nabla^{\prime} P^{\prime}+\rho \mathbf{g}^{\prime}+\mu^{\prime} \nabla^{\prime} \cdot\left(\frac{\nabla^{\prime} \mathbf{u}^{\prime}+{ }^{t} \nabla^{\prime} \mathbf{u}^{\prime}}{2}\right)+\gamma^{\prime} \kappa^{\prime} \delta_{s}^{\prime} \mathbf{n}
$$

where the primes represent dimensionless variables.

\section{Simulated cases and conditions}

We performed numerical simulations for typical raindrop impacts falling on a water layer. All computations were done for spherical raindrops of diameter equal to $D=2 \mathrm{~mm}$. Considering the scaling factor $D^{\prime}=0.3$, this leads to a domain of $6.67 \mathrm{~mm}$ in both width and height. The raindrop velocity was set to its terminal velocity, $U_{0}=6.5 \mathrm{~m} \mathrm{~s}^{-1}$. The thickness of the water film $h$ varied from $D / 10$ (i.e $0.2 \mathrm{~mm}$ ) to $2 D$ (i.e. $4 \mathrm{~mm}$ ), with the intermediate cases $D / 5$, $D / 3, D / 2$ and $D$.

Standard air and water properties were used: $\rho_{l}=10^{3} \mathrm{~kg} \mathrm{~m}^{-3}, \mu_{l}=$ $10^{-3} \mathrm{~kg} \mathrm{~m}^{-1} \mathrm{~s}^{-1}, \rho_{g}=1 \mathrm{~kg} \mathrm{~m}^{-3}, \mu_{g}=2 \times 10^{-5} \mathrm{~kg} \mathrm{~m}^{-1} \mathrm{~s}^{-1}$, with a surface tension $\gamma=0.02 \mathrm{~kg} \mathrm{~s}^{-2}$. In this configuration, the Reynolds number $\operatorname{Re}$ was 6500 and the Weber number We 2112.5. These large values indicate that inertia dominates a priori the other forces. Preliminary testing confirmed that the effect of gravity was negligible during a raindrop impact. Consequently, gravity was not included in the simulations.

At high velocities, drop impacts develop angular instabilities leading to the famous pictures of splashing, popularized for instance in commercials. Splash is one of the key issue of drop impacts identified already by Worthington (1876) in the first studies on drop impacts, leading for instance to secondary droplet breakups (Rein, 1993). These splashing dynamics can be important in soil erosion because it can transport eroded material at large distances as shown by 
Planchon and Mouche (2010). In the present case, the axisymmetric geometry can be used because 1) we are focusing on the erosion mechanism itself and not on the transport of particle, and 2) such instabilities become relevant for time scales much larger than the typical time scale of the pressure and shear stress development at the soil surface. Consequently, an axial boundary condition was imposed on the symmetry axis $\left(r^{\prime}=0\right)$. At the soil surface $\left(z^{\prime}=0\right)$, a zero velocity boundary condition (also known as Dirichlet condition) was set. This ensured that both 1) no infiltration $\left(u_{z}^{\prime}=0\right)$ and 2) no slip $\left(u_{r}^{\prime}=0\right)$ occurred. For the top $\left(z^{\prime}=H_{\text {max }}^{\prime}=1\right)$ and radial $\left(r^{\prime}=R_{\text {max }}^{\prime}=1\right)$ boundaries, either Neumann (no slip) or Dirichlet (zero velocity) boundary conditions could potentially be used. Preliminary testing showed that the type of boundary condition did not influence the results because the simulated domain was large enough compared to the area of interest. For the simulations, a Dirichlet condition was used at $z^{\prime}=H_{\max }^{\prime}$ and $r^{\prime}=R_{\max }^{\prime}$.

During the simulation of a raindrop impact, the water height $h$ can become zero (especially for thin initial water depths). The occurrence of cells with $h=0$ requires special attention, because it involves the motion of the contact line separating the water and the air along the soil surface (i.e. a triple-point occurs). In general, a specific boundary condition should be applied at the moving contact point to account for the high viscous stresses involved (Afkhami et al., 2009). In our case, an alternative approach can be used by acknowledging that a real soil surface is not exactly smooth but involves some roughness that can be crucial for the dynamics of the impact. This roughness can be taken into account by imposing a Navier slip boundary condition on the soil surface with a slip length of the order of the roughness (Barrat and Bocquet, 1999). Technically, since the usual no-slip boundary condition imposed by the numerical scheme corresponds to a Navier slip condition with a slip length of the order of the mesh size, one has to simply take the no-slip boundary condition here with a mesh size similar to the surface roughness. Therefore, the numerical no-slip boundary conditions imposed for a constant level of refinement can be interpreted as a natural model for the soil roughness. In that framework, throughout the simulations, we can consider that a surface roughness equal to $65 \mu \mathrm{m}$ was used (level of refinement $n=10)$.

\section{Results and discussion}

\section{Overall dynamics}

The phenomenology of a drop impact on a thin water layer is illustrated for the case $h=D / 10$ in Figure 2, where the interface, the velocity and the pressure 
fields are shown together for different times. In the following, the initial time $t^{\prime}=0$ is taken as the theoretical time of impact, defined by the falling velocity $U_{0}^{\prime}=1$ of a sphere onto the undeformed flat liquid layer.

At $t^{\prime}=10^{-3}$ (i.e. $1 \mu \mathrm{s}$ after the impact initiation), the drop and the water layer are still separated by a narrow sheet of air (Figure 2a). Nevertheless, the pressure has started to increase in the water, mediated by the high lubrication pressure created in the cushioning air layer located between the drop and the liquid film. The maximum pressure $\left(P_{\max }^{\prime}=1.85\right.$, i.e. $\left.P_{\max }=78.2 \mathrm{kPa}\right)$ is between the drop and the water layer.

At $t^{\prime}=10^{-2}$ (i.e. $10 \mu \mathrm{s}$ after the impact initiation), the drop and the water layer have started to merge and some air is trapped inside the water (Figure $2 \mathrm{~b}$ ) due to the air cushioning (Thoroddsen et al., 2003, Korobkin et al., 2008). A high pressure field is created, with a maximum pressure of $P_{\max }^{\prime}=2.68$ (i.e. $P_{\max }=$ $113.2 \mathrm{kPa}$ ) now located close to the wedge formed by the intersection between the drop and the liquid layer. At $t^{\prime}=0.03$ (i.e. $31 \mu \mathrm{s}$ after the impact initiation), most of the water which belonged to the raindrop still have its terminal velocity (Figure 2c). It is only in the impact region that the velocity vectors rotate from the vertical. In this same area, the velocities are smaller than the terminal velocity but in the small wedge region one can see the formation of a high-speed jet created by the high pressure peak. Indeed, the maximum pressure is still located near the wedge but has started to decrease $\left(P_{\max }^{\prime}=1.47\right.$, i.e. $\left.P_{\max }=62.1 \mathrm{kPa}\right)$. A few droplets are emitted from the wedge.

At $t^{\prime}=0.08$ (i.e. at $t=82 \mu \mathrm{s}$ ), a complex velocity field is formed (Figure 2d). Firstly, a jet has been emitted by the impact, leading to a splash of which the specific dynamics would be fully three dimensional and which is not at the heart of the present study. Secondly, close to the soil surface, the velocity field is expanding mostly radially due to the spreading of the raindrop into the water layer. Together with the no-slip boundary condition on the soil surface, it leads to a radial velocity field depending both on the radius $r^{\prime}$ and the vertical coordinate $z^{\prime}$. In fact, the no-slip boundary condition imposed at $z^{\prime}=0$ induces the formation of a viscous boundary layer between the substrate and the radial flow created by the impact (Roisman, 2009, Eggers et al., 2010). Hence, the soil is subjected to a significant shear stress which is crucial for erosion processes. The pressure field is now maximum near the soil surface, directly under the impact region, but its maximum value has decreased to $P_{\max }^{\prime}=0.8$ (i.e. $P_{\max }=$ 33.8 kPa).

This general description is in agreement with the previous publications on raindrop impacts on a water layer (Wang and Wenzel, Jr, 1970, Ghadiri and Payne, 1977, 1980, 1986; Hartley and Alonso, 1991; Hartley and Julien, 1992; Marengo et al., 2011).

Since the erosion rate depends mostly on the shear stress applied on the soil 


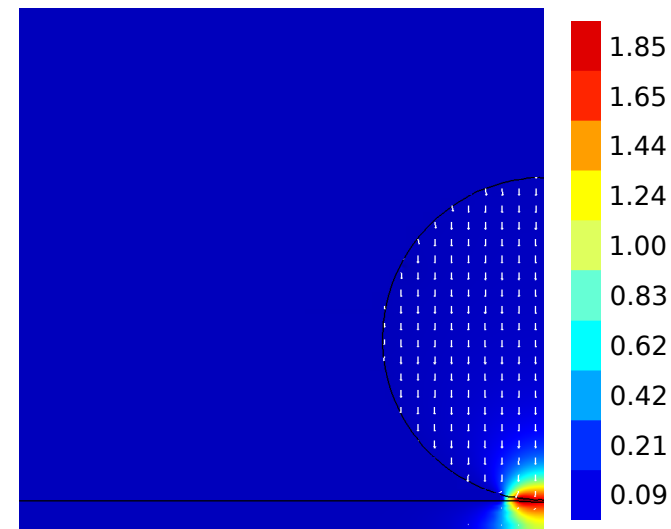

(a) $t^{\prime}=10^{-3}$ (i.e. $1 \mu$ s)

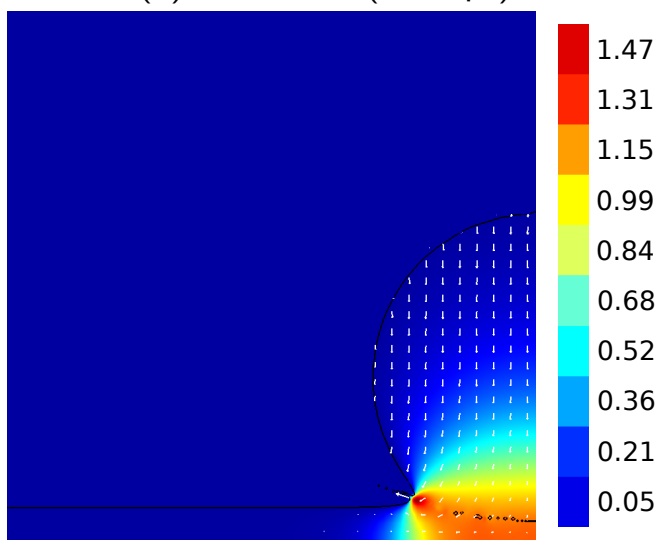

(c) $t^{\prime}=0.03($ i.e. $31 \mu \mathrm{s})$

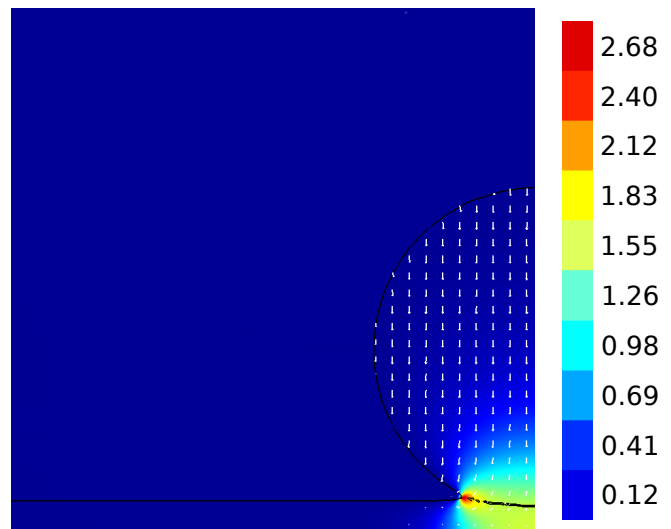

(b) $t^{\prime}=10^{-2}$ (i.e. $\left.10 \mu \mathrm{s}\right)$

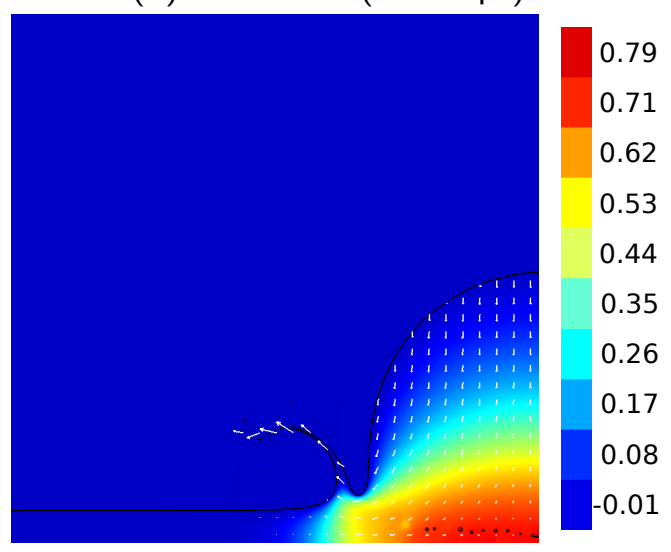

(d) $t^{\prime}=0.08$ (i.e. $\left.82 \mu \mathrm{s}\right)$

Figure 2: Pressure and velocity fields in the water (colors and arrows respectively) as function of time for a film layer of $h=\frac{D}{10}$.

The drop diameter is $2 \mathrm{~mm}$ with a terminal velocity of $6.5 \mathrm{~m} \mathrm{~s}^{-1}$. 
surface, a detailed analysis of the dynamical evolution of the stress tensor during the impact is needed. In particular, the Meyer-Peter and Müller equation is often used, relating the erosion rate $q_{s}$ to the shear stress $\tau$ through (Meyer-Peter and Müller, 1948; Houssais and Lajeunesse, 2012):

$$
q_{s}^{\prime}=c\left(\tau^{\prime}-\tau_{c}^{\prime}\right)^{3 / 2}
$$

where the dimensionless erosion rate and shear stress are defined by:

$$
q_{s}^{\prime}=\frac{q_{s}}{\sqrt{\left(\rho_{s} / \rho_{l}-1\right) g d^{3}}} \text { and } \tau^{\prime}=\frac{\tau}{\left(\rho_{s}-\rho_{l}\right) g d}
$$

where $d$ is the typical size of the grains composing the soil, $\rho_{s}$ its density and $c$ an empirical constant fitted through experimental data. In the following, we will use the numerical simulations done for raindrop conditions to deduce scaling laws for the shear stress induced by the impact that we will compare with simple formulas obtained using a self-similar model. Prior to the shear stress itself, we will investigate the pressure field created by the impact, where self-similar behavior has already been observed (Josserand and Zaleski, 2003). Here, selfsimilarity means that the pressure field depends only on a quantity that is time dependent. In particular, it means that the pressure field conserves the same shape with time, with only amplitude and size varying with time.

\section{Pressure evolution inside the water and self-similar approach}

In fluid mechanics, scaling laws have been deduced from numerical simulations of the pressure evolution inside the water during the impact of a droplet on a solid surface or in the limit of thin liquid films, using a self-similar approach (Josserand and Zaleski, 2003: Eggers et al., 2010). However, their validity has not been studied in the context of raindrop impacts yet, in particular when the liquid film thickness varies. The self-similar approach is based on a theory first developed by Wagner (1932) using as the typical length scale involved in the impact, the intersection between a falling spherical drop and the unperturbed liquid layer surface. In other words, the pertinent length scale of the impact $r_{c}(t)$ (or $r_{c}^{\prime}\left(t^{\prime}\right)$ in dimensionless form) follows:

$$
r_{c} \propto \sqrt{D U_{0} t} \text { or } r_{c}^{\prime} \propto \sqrt{t^{\prime}}
$$

where $t\left(t^{\prime}\right)$ is the time after the contact of the falling drop on the surface. This formula corresponds to the intersection of the drop (taken as a circle of radius $D / 2$ ) that is in contact with the water surface at time $t=0$. The selfsimilar theory takes advantage of the observation on the numerical simulations 
that the perturbed region of the drop at short time after impact is governed by $r_{c}$ (Josserand and Zaleski, 2003). Such self-similar approach is possible when no specific length scale is dominating the dynamics: this is precisely the situation at short time for high Reynolds and Weber numbers, where only the intersection between the falling drop and the impacting liquid layer is thus playing a role.

Indeed, figure 3 shows the evolution with time of the radial position $r_{c}^{\prime}$ of the maximum pressure in the water for different liquid layer thicknesses. This evolution can be separated into three stages. For $t^{\prime}<2 \cdot 10^{-3}$ (i.e. for durations smaller than $2.1 \mu \mathrm{s})$, the evolution of $r_{c}^{\prime}$ depends on the ratio $h / D$. This is also true for $t^{\prime}>2.10^{-2}$ (i.e. for durations larger than $20.5 \mu \mathrm{s}$ ), where it can also be noticed that, at the beginning of this period, $r_{c}^{\prime}$ is of the order of one raindrop radius (i.e. $r_{c}^{\prime}=0.15$ in Figure 3 ). In the intermediate stage, all the values of $r_{c}^{\prime}$ collapse onto a single straight line (in log-log scale), meaning that the relationship between the location of the maximum pressure and time is independent of the ratio $h / D$. Over this period, the position of the maximum pressure $r_{c}^{\prime}\left(t^{\prime}\right)$ is in good agreement with the former square-root law (6), yielding quantitatively:

$$
r_{c}^{\prime}=0.65 \sqrt{t^{\prime}}
$$

Remarkably and as predicted by the theory, this law is independent of the layer thickness, in addition to being found independent on the Reynolds and Weber numbers in previous studies (as long as these numbers are high enough) (Thoroddsen, 2002, Josserand and Zaleski, 2003).

As geometrically deduced, this geometric law should not be valid for $r_{c}>$ $D / 2$. However, it is well known that the square-root law for $r_{c}$ is in fact observed for much larger values and in the figure, the law is typically valid up to $r_{c} \sim 2 D$. Indeed, it has been argued that such a square-root law is also the cylindrical shock solution of the shallow-water equations as explained in Yarin and Weiss (1995) so that the geometric law matches this shock solution for longer times. The limitation of this regime at short and long times can be explained by two distinct arguments. At short time, the cushioning of the air layer delays the contact between the drop. At long time scales, numerical limitations can also be present: because the drop spreading has a large spatial extent, finite size effects coming from the size of the numerical box start to affect the dynamics.

Similar regimes are observed for the maximum pressure in the water $P_{c}^{\prime}$ as shown in Figure 4 . Firstly, the pressure is slightly varying at short time $t^{\prime}<$ $2.10^{-2}$ (i.e. lower than $20.5 \mu \mathrm{s}$ ) and does not depend on the $h / D$ ratio. At this stage the contact between the raindrop and the water layer is weak and we attribute this effect to the lubrication pressure created in the gas layer. At long time, typically corresponding to $r_{c}^{\prime}>D / 2$, the pressure is rapidly dropping to very small values. In between, corresponding roughly to $2 \cdot 10^{-2} \leq t^{\prime} \leq 10^{-1}$ 


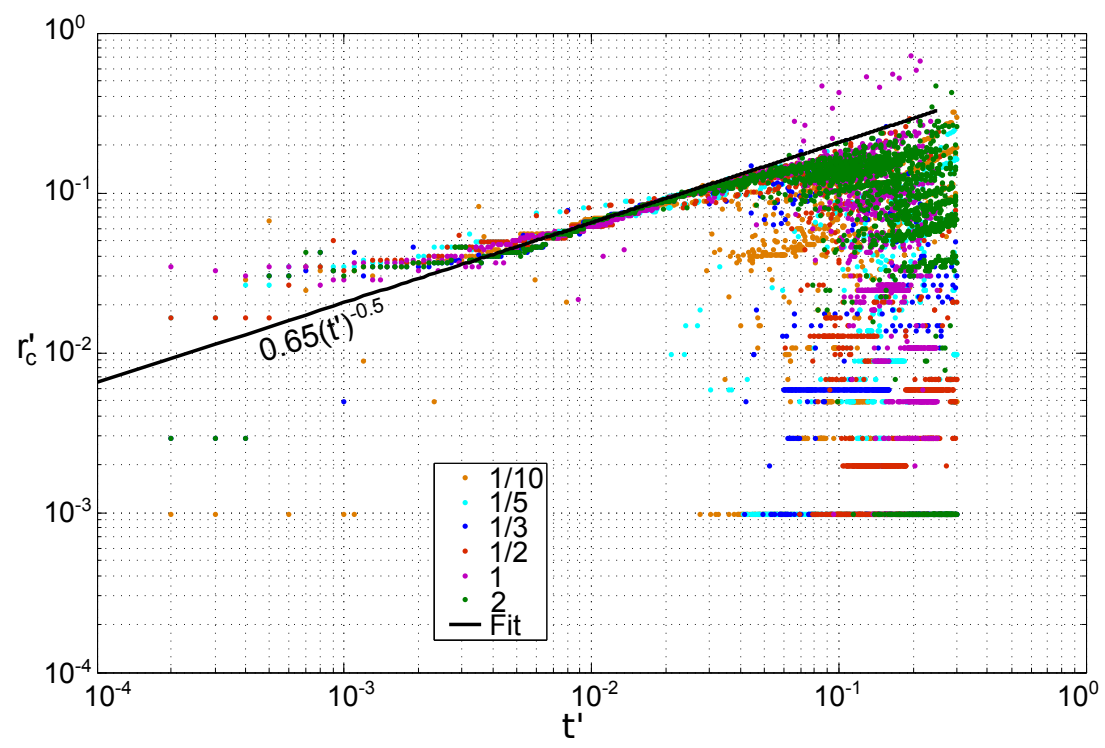

Figure 3: Position of the maximal dimensionless pressure as function of time after impact for different water depth/drop diameter ratios.

Note: the exponent was not fitted.

the pressure is decreasing with time following power law behaviors. However, two distinct regimes are observed depending on the ratio $h / D$ : for small aspect ratio (typically $h / D<1 / 4$ ) the maximum pressure is found to decrease following the inverse of the square-root of the time (Figure 4 a), as usually observed for the impact on thin liquid layer (Josserand and Zaleski, 2003). For thicker water layers (i.e. for $h / D \geqslant 1 / 4$ ), another regime is observed where the maximum pressure decreases first like the inverse of time (Figure $4 \mathrm{~b}$ ), while the inverse of the square-root of the time seems to remain valid at larger time. The crossover between these two time dependances increases with the aspect ratio $h / D$.

As detailed in Josserand and Zaleski (2003), the thin water layer behavior can be understood using a simple momentum balance in the self-similar impact region. Indeed, it has been observed that in this regime, the pressure field is perturbed in the impacted region only, defined by the characteristic length $r_{c}(t)$. Therefore, one can develop a self-similar approach using this length and perform the vertical momentum balance in the self-similar volume of radius $r_{c}(t)$ (the volume being that of a half sphere of radius $r_{c}$, namely $2 \pi r_{c}^{3} / 3$ ), yielding:

$$
\frac{d\left(2 \rho_{l} \pi r_{c}(t)^{3} U_{0} / 3\right)}{d t} \sim \pi r_{c}(t)^{2} P_{c}(t),
$$

where $P_{c}(t)$ is the typical amplitude of the pressure field created by the impact. This equation balances the variation of vertical momentum in the self-similar 


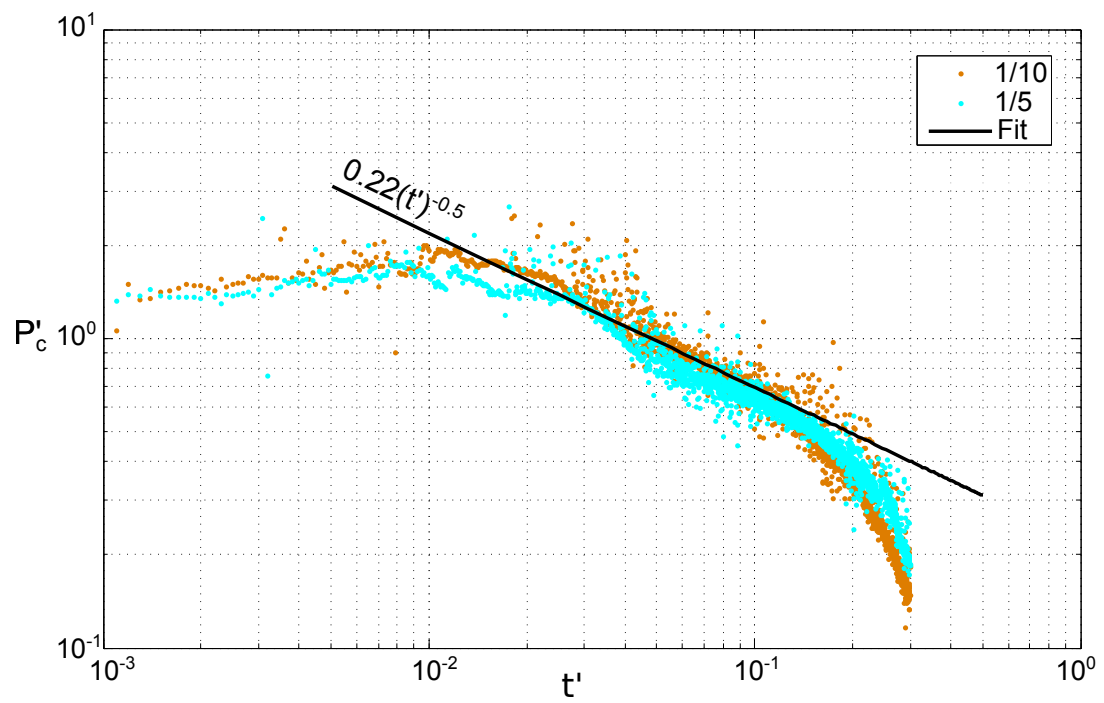

(a)

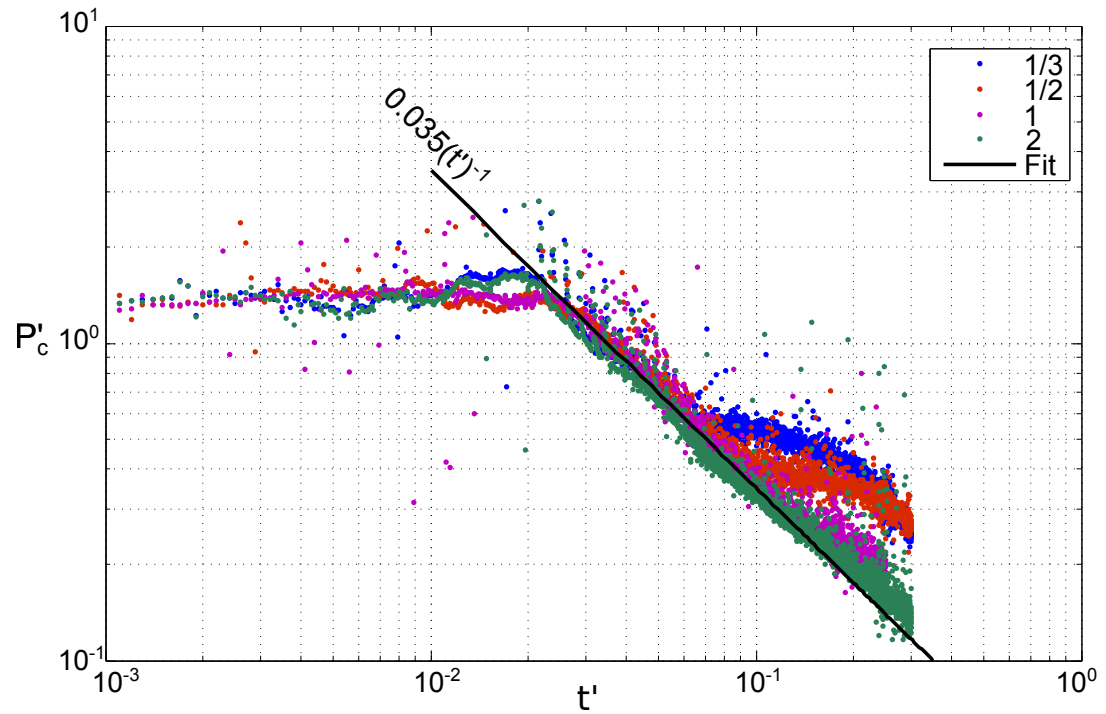

(b)

Figure 4: Maximum dimensionless pressure as function of time for (a) small and (b) large water depth to drop diameter ratios. Note: the exponents were not fitted. 
half-sphere of radius $r_{c}(t)$ with the force of the pressure on the liquid layer, giving thus:

$$
P_{c}(t) \propto \rho_{l} U_{0} \frac{d r_{c}(t)}{d t} \sim \rho_{l} U_{0}^{2} \sqrt{\frac{D}{U_{0} t}} .
$$

This regime is in good agreement with the observed maximum pressure evolution for thin liquid films (Figure 4a). Moreover, this regime start to fail at longer time (typically corresponding to $\left.r_{c}(t) \sim D / 2\right)$ and the dynamics can then be described by the shallow-water equation (Yarin and Weiss, 1995: Lagubeau et al., 2010).

For thick water layers, the former vertical momentum balance does not work since the liquid layer dynamics have to be taken into account. However, inspired by the former balance, one can deduce a simple model: considering that the radial characteristic length is still $r_{c}$ but that the vertical one is now $h$, we obtain

$$
\frac{d\left(\rho_{l} \pi r_{c}(t)^{2} h U_{0}\right)}{d t} \sim \pi r_{c}(t)^{2} P_{c}(t)
$$

which gives the observed scaling for the pressure:

$$
P_{c}(t) \sim \rho_{l} U_{0} \frac{h}{r_{c}(t)} \frac{d r_{c}(t)}{d t} \sim \rho_{l} U_{0}^{2} \frac{h}{U_{0} t} .
$$

The former thin layer regime is retrieved at larger time in this configuration and one can argue that it comes from the fact that the liquid contained in the layer has been pushed away by the impact so that only a thin residual liquid layer remains beneath the drop.

Therefore, we have exhibited that the pressure field due to the impact follows self-similar laws involving the spreading radius $r_{c}(t)$. However, as explained above, the crucial quantity for the erosion process is not the maximum pressure in the liquid but rather the shear stress at the soil surface. The self-similar approach can be used a priori to compute the soil surface quantities, but one has to notice that the soil surface does not coincide with the self-similar geometry (which involves the liquid layer interface rather than the solid surface). In conclusion it leads to the difficult challenge of determining the shear stress at the intersection between the self-similar geometry and the soil surface. Moreover, the shear stress is also a consequence of the boundary layer created by the large scale flow and the no slip boundary condition imposed at the surface (Roisman, 2009: Eggers et al., 2010 Lagubeau et al., 2010), which makes its prediction even more difficult.

\section{Scaling laws for stresses onto the soil surface}

We thus now investigate the pressure and shear stress fields onto the soil surface, quantities of interest for understanding and modeling erosion processes, keeping 
in mind the underlying self-similar structure of the impact.

\section{Dependency of the maximum shear stress with the water depth}

First of all, let us mention that the dependence of the shear stress with the water depth has already been studied using numerical simulations by Hartley and Alonso (1991) and Hartley and Julien (1992), leading to an algebraic fitted relationship for the maximum (over time and space) shear stress at the soil surface, as a function of the Reynolds number and the water depth:

$$
\tau_{\max }=2.85 \rho_{l} U_{0}^{2}\left(\frac{h}{D / 2}+1\right)^{-3.16} \operatorname{Re}^{-0.55} C_{1}
$$

The prefactor $C_{1}$ is almost a constant number varying only slightly with the impact parameters between 0.91 and 1 and deviating from one only for slow drops and thick water layers. However, this relationship was based on simulations that included only Reynolds numbers within the range 50-500 and Weber numbers in the range 18-1152 (Hartley and Alonso, 1991; Hartley and Julien, 1992), values much lower than the range of natural raindrops $(6500 \leqslant R e \leqslant 23000$, $50 \leqslant W e \leqslant 12150)$. Hence, their simulations underestimated the inertia forces compared to both viscous and capillary forces.

In the present study, we have performed numerical simulations for realistic $R e$ and We numbers for raindrops, varying only the water depth. Figure 5 shows the maximum shear stress $\tau_{\max }$ as a function of the liquid depth plus the drop radius normalized by the drop radius, which can be written as: $1+2 h / D$. It is well fitted by the following relationship:

$$
\tau_{\max } \propto\left(\frac{h}{D / 2}+1\right)^{-2.6}
$$

The maximum shear stress is observed around the time $(D / 2+h) / U_{0}$ that would correspond to the penetration of half of the unperturbed drop over all the liquid layer. This relationship was fitted only varying the ratio $h / D$ so that the physical prefactor involves $\rho_{l} U_{0}^{2}$ multiplied by some function of the dimensionless numbers (in particular the Reynolds number). As the aspect ratio $h / D$ is concerned, we observed that our fitted law is slightly different than the Hartley law (7), the exponent of the fraction being smaller. However, given the variation of $1+2 h / D$ studied here, one should remark that the quantitative differences between our results and those of Hartley are not very large. In order to suggest an explanation for such dependence, a detailed study of the evolution of the stress tensor at the soil surface is first needed. 


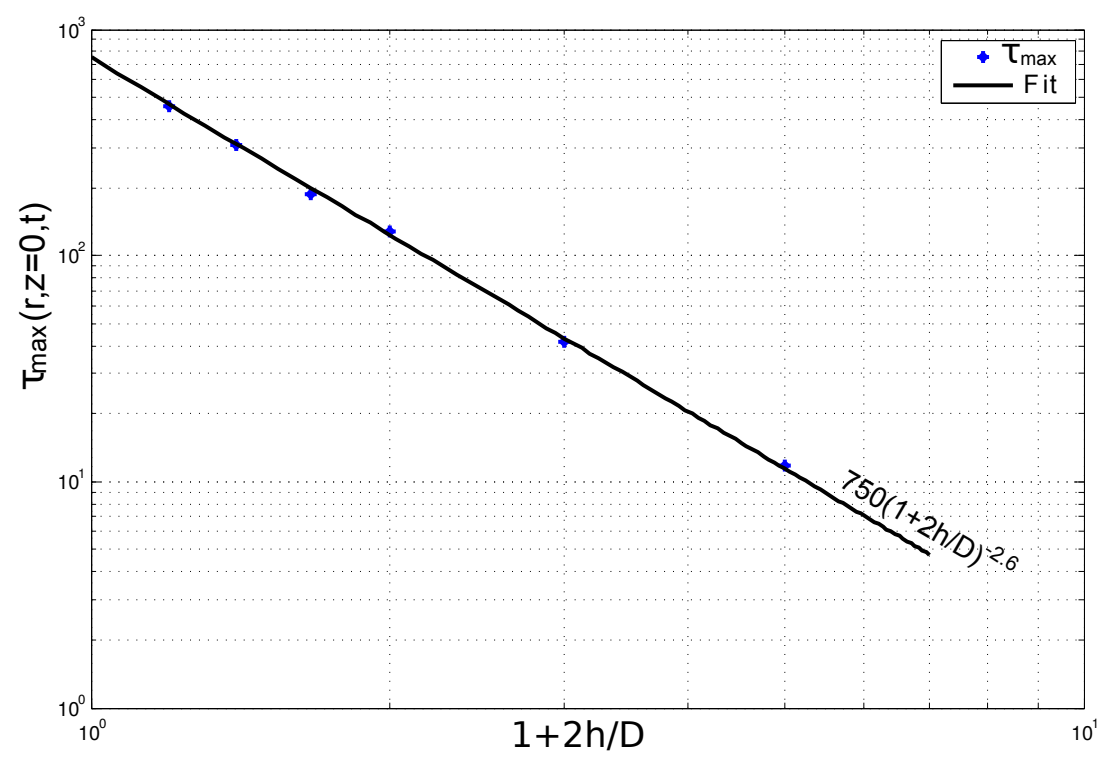

Figure 5: Maximum physical shear stress at the soil surface as a function of $(1+2 h / D)$.

\section{Self-similar evolution of the stress tensor on the soil surface Pressure}

The pressure field on the soil surface shows a bell-shape curve with a maximum on the symmetry axis $(r=0)$ for all times, the amplitude of this curve decreasing with time while its width increases. We thus define the characteristic length for the pressure field on the soil surface as the radius where the pressure is half the pressure on the axis $r_{P_{1 / 2}}^{\prime}(t)$. The width $r_{P_{1 / 2}}^{\prime}\left(t^{\prime}\right)$ is shown in Figure 6 as a function of time for different aspect ratios $h / D$. For short time after impact, the evolution of $r_{P_{1 / 2}}^{\prime}(t)$ can be fitted by a power-law, the exponent $m$ decreasing with the aspect ratio. For the thinnest simulated water layers, $r_{P_{1 / 2}}^{\prime}(t)$ evolves as the square-root of $t^{\prime}(m \sim 0.5)$ which is consistent with the law obtained for thin films. The exponent $m$ of the power-law decreases when the water layer increases (see insert of Figure 6) Thus the water layer can be seen as a shield protecting the soil surface against the disturbance caused by raindrop impacts and it is only for the thinnest water layers, i.e. when the shielding is the lowest, that the disturbance (here the pressure) is similar inside the water layer and at the soil surface.

For deeper water layers, the shielding is more efficient, leading to a disconnection between the behavior of the pressure inside the liquid and the behavior of the pressure at the soil surface. This disconnection becomes quite significant 


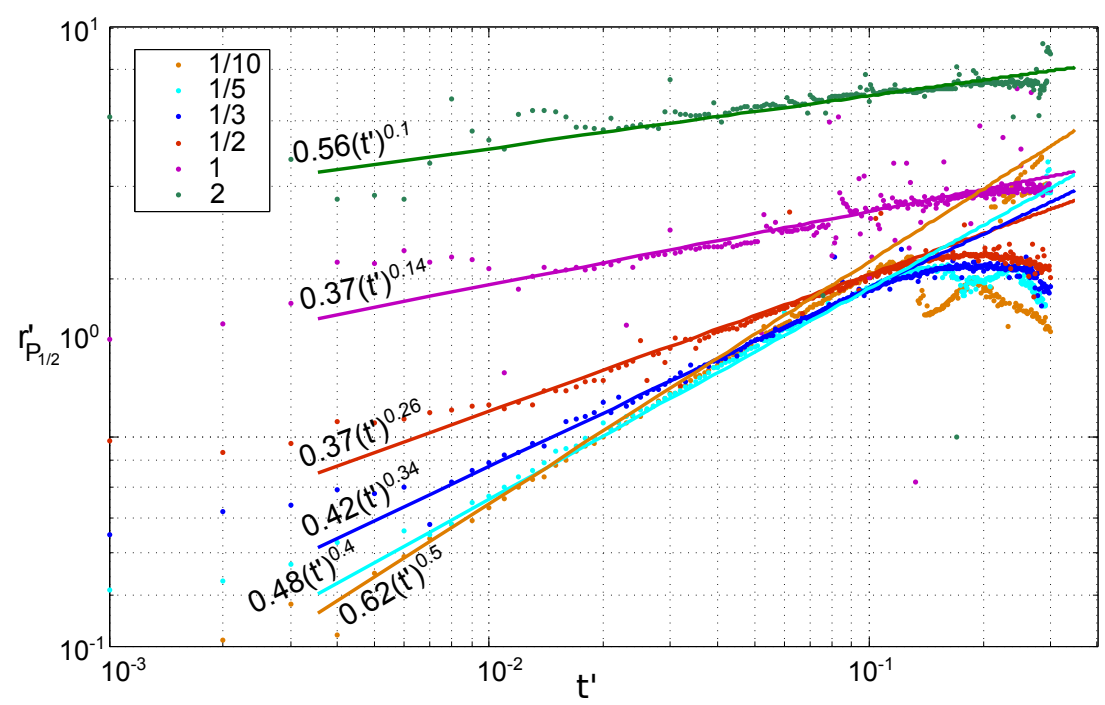

Figure 6: Dimensionless radial position $r_{P_{1 / 2}}^{\prime}\left(t^{\prime}\right)$ of half the maximum dimensionless pressure (located at $r=0$ ) at the soil surface for different ratios $h / D$ shown on insert. The fitted power-law is traced on the figure for each value of $h / D$.

for a water layer equal to the drop radius.

However, a self-similar structure of the pressure field on the soil surface can also be exhibited for the different aspect ratios $h / D$. Indeed, rescaling the pressure on the soil surface $P^{\prime}\left(r^{\prime}, z^{\prime}=0, t^{\prime}\right)$ at different times by the maximum value $P^{\prime}\left(0,0, t^{\prime}\right)$ and the coordinate $r^{\prime}$ by $r_{P_{1 / 2}}^{\prime}(t)$, we observe a good collapse of the different pressure curves into a single one for $t^{\prime}<10^{-1}$ (i.e. smaller than 102.6 $\mu \mathrm{s}$ ) (Figure 7). However, these self-similar curves vary with the aspect ratio $h / D$ (Figure 8), the width $e$ of the self-similar curve increasing with $h / D$.

\section{Shear stress}

The shear stress at the soil surface is computed numerically using the shear rate, yielding:

$$
\tau=\mu_{l} \frac{d u_{r}(r, z=0)}{d z} .
$$

and it exhibits a ring shape of which the radius $r_{\tau_{\max }}^{\prime}\left(t^{\prime}\right)$, corresponding to the maximum shear stress location, increases with time. For thin liquid layers, $r_{\tau_{\max }}^{\prime}(t)$ evolves again approximately as the square-root of $t^{\prime}$ again (Figure 9a) while the situation is more complex for $h / D \geqslant 1$, where no tendency could be extrapolated (Figure 9b).

We rescaled again the dimensionless shear stress $\tau^{\prime}(r, z=0, t)$ by its maximum value (noted $\tau_{\max }^{\prime}(r, z=0, t)$ ) and plotted it as a function of the rescaled 


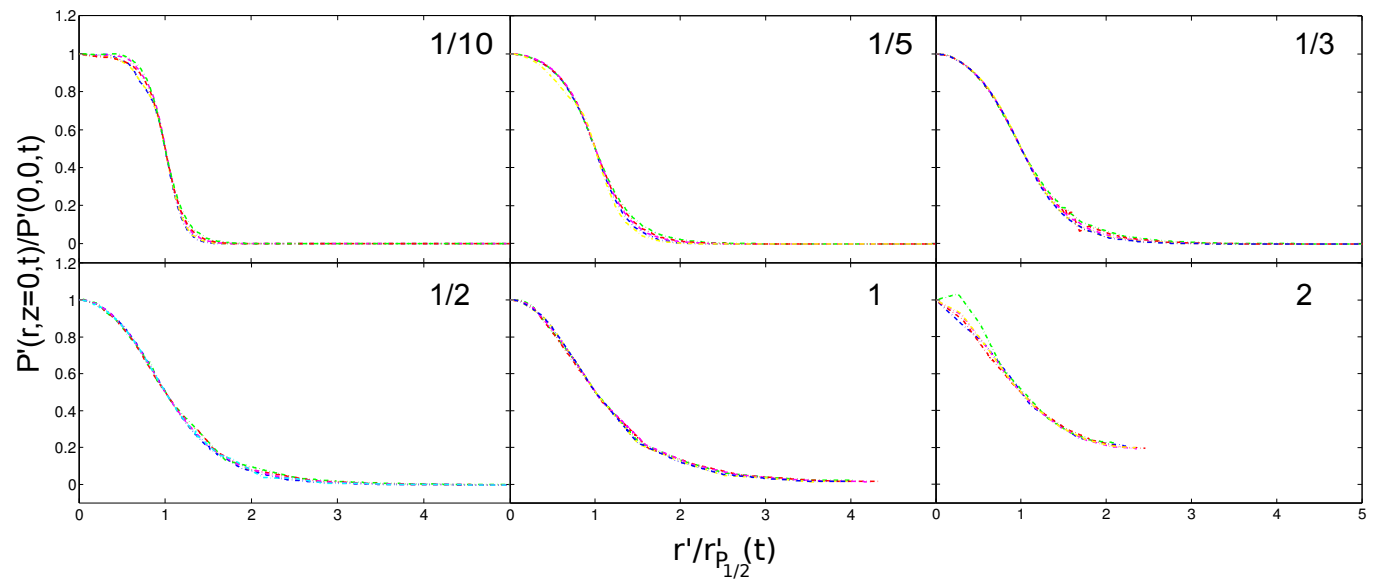

Figure 7: Rescaled pressure on the substrate for different aspect ratios $h / D$ (indicated on each figure) for $t^{\prime}<10^{-1}$.

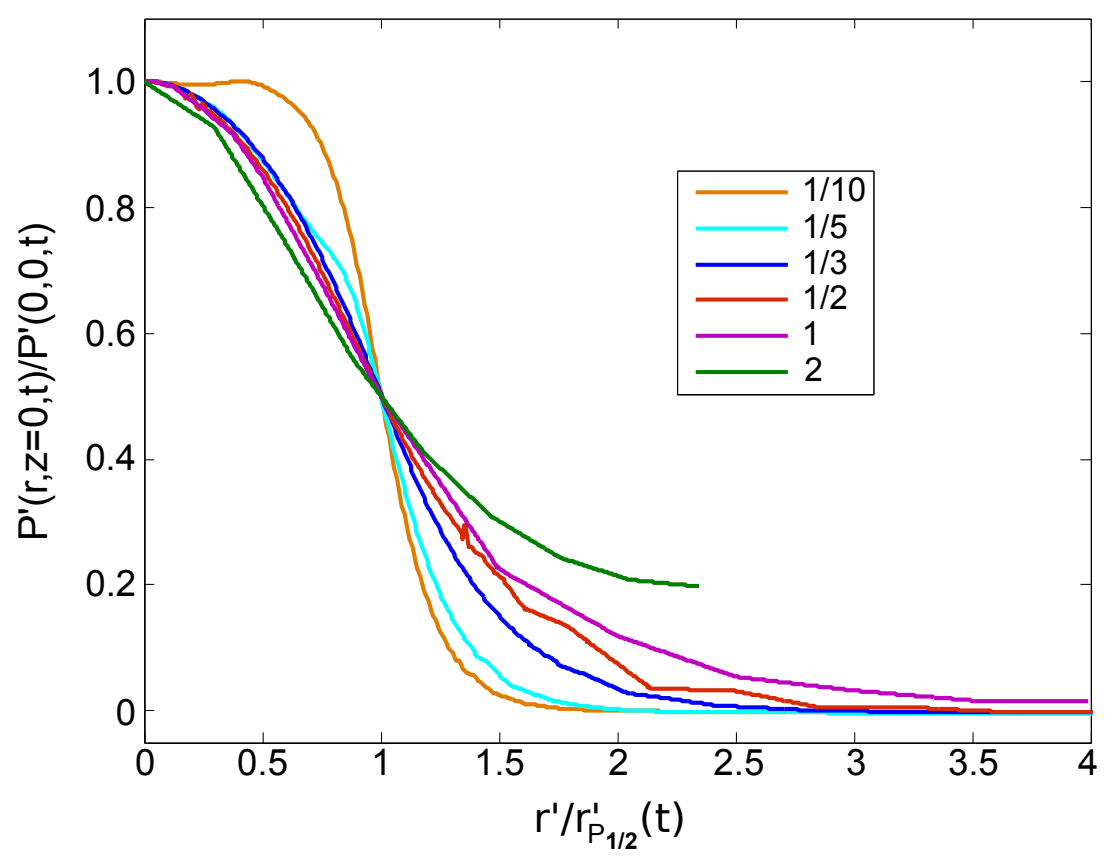

Figure 8: Comparison of the rescaled pressure for different aspect ratios $h / D$. 


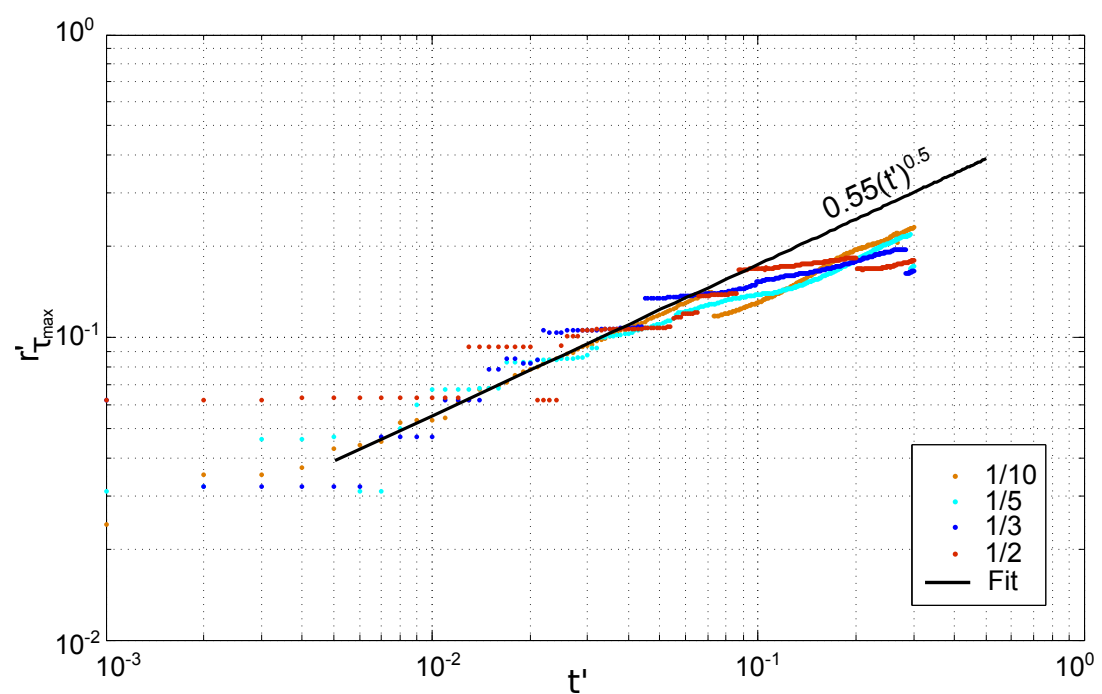

(a)

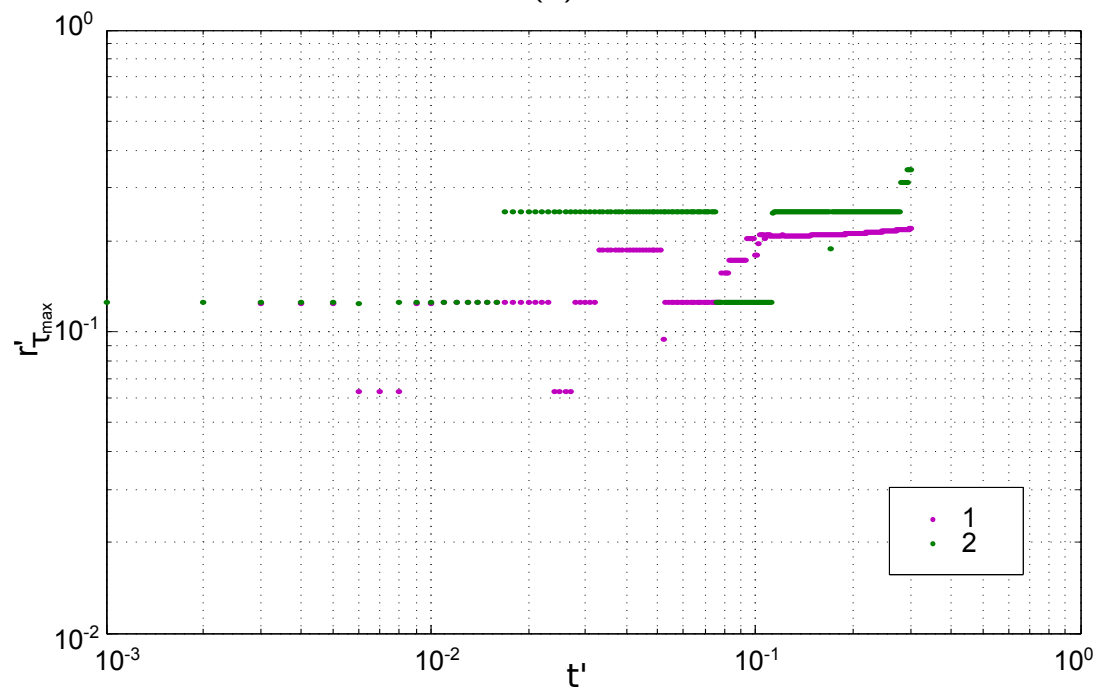

(b)

Figure 9: Time evolution of the dimensionless radial position $r_{\tau_{\max }^{\prime}(t)}$ of the maximum dimensionless shear stress at the soil surface for (a) small and (b) large $h / D$.

Note: the exponent was not fitted. 
radius $r^{\prime} / r_{\tau_{\max }}^{\prime}(t)$ (where $r_{\tau_{\max }^{\prime}}(t)$ is the position of the maximum value of $\tau^{\prime}$ at $t$ ) for small time $t^{\prime}<10^{-1}$ (i.e. smaller than 102.6 $\mu \mathrm{s}$ )(Figure 10). The collapse of the profiles is reasonable for small $(<1 / 3)$ and large $(>1)$ aspect ratios $h / D$ but the situation is much more complex for intermediate cases where only a partial collapse is found. The shear stress has a quasi-linear profile for small radius $\left(r^{\prime}<r_{\tau_{\max }}^{\prime}(t)\right)$ and then relaxes to zero for large $r^{\prime}$. Such behavior for small radius can be understood within the dynamics of thin films (Yarin and Weiss, 1995, Lagubeau et al., 2010). This regime is valid after the self-similar regime of the impact for which the pressure is high. Then, assuming a small gradient of the interface, the dynamics follows the so-called thin film equations for which the radial velocity yields:

$$
u=\frac{r^{\prime}}{t^{\prime}}
$$

for $r^{\prime}<r_{\tau_{\max }}^{\prime}(t)$. This radial velocity is not consistent with the no-slip boundary condition on the $z=0$ solid boundary so that a viscous boundary layer of thickness $I_{v}=\sqrt{\mu t / \rho}$ grows from the solid (Roisman, 2009, Eggers et al. 2010). Therefore, one obtains for the shear stress on the solid (in dimensionless form):

$$
\tau^{\prime}(r, z=0, t) \propto \frac{r^{\prime}}{t^{\prime 3 / 2} R e^{1 / 2}},
$$

which is consistent with the linear behavior for small $r^{\prime}$. On the other hand, this regime can also explain the dependence of the maximum shear stress. Indeed, assuming that for the maximum shear stress obtained at time $t^{\prime}=1+2 H / D$, the radial momentum of the thin film is equal to the vertical momentum of the impacting drop, one obtains approximately:

$$
D^{3} U_{0} \sim h_{c} D^{2} U_{0} \frac{\left(r_{\tau_{\max }^{\prime}}^{\prime}\right)^{3}}{t_{\tau_{\max }}^{\prime}}
$$

where $h_{c}$ is the film height in the impacted zone, a priori different than the unperturbed film height $h$. Then, we obtain, taking the time of maximum shear stress at $t_{\tau_{\max }}^{\prime} \propto 1+2 h / D$

$$
\frac{r_{\tau_{\max }}^{\prime}}{1+2 h / D} \sim \frac{1}{\left(r_{\tau_{\max }}^{\prime}\right)^{2} h_{c} / D}
$$

Then using the observed relation $r_{\tau_{\max }}^{\prime} \propto \sqrt{t_{\tau_{\max }}^{\prime}}$, we have

$$
\tau_{\max }^{\prime} \propto \frac{1}{(1+2 H / D)^{3 / 2} h_{c} / D} R e^{-1 / 2} .
$$


Assuming the same scaling relation for the film height $h_{c} / D \propto 1+2 h / D$ we obtain

$$
\tau_{\max }^{\prime} \propto \frac{1}{(1+2 H / D)^{5 / 2}} R e^{-1 / 2}
$$

which is in good agreement with the numerical results. Note that the exponent $5 / 2$ is obtained from assumptions that are very speculative and which would need further studies to validate. However it is very close to the 2.6 fitted exponent of relation (8), shedding light on the underlying mechanism for the film thickness that is at play in the shear stress formula. In particular, this exponent combines the contribution of the thin film velocity field with the viscous boundary layer. The $R e^{-1 / 2}$ is a direct consequence of the boundary layer structure and has thus better scientific grounds although it has not been tested in our numerics. Notice that it is in good agreement with the previous observed behavior (Hartley and Alonso, 1991; Hartley and Julien, 1992). We would like also to emphasize that such analytical formula is very important since it could be implemented in macroscopic models coupling raindrop and erosion.

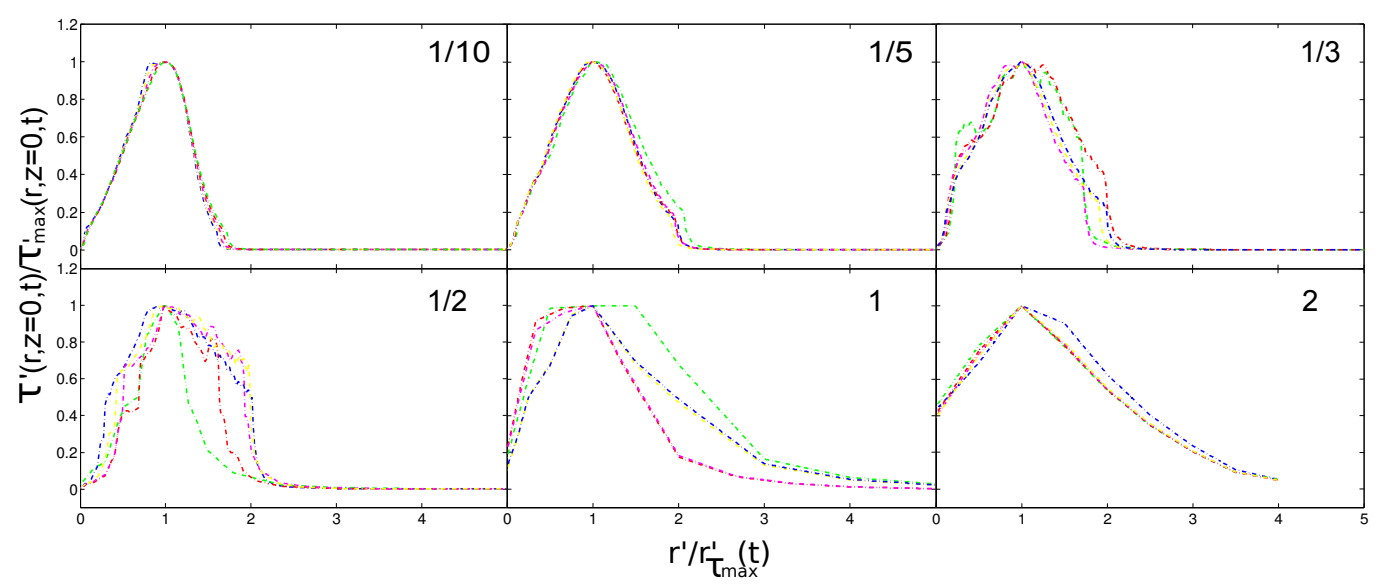

Figure 10: Rescaled dimensionless shear stress for different aspect ratios $h / D$ for $t^{\prime}<10^{-1}$.

\section{Conclusions}

Using present numerical methods to solve the Navier-Stokes equations for liquidgas dynamics, we have studied raindrop impacts on water layers for realistic configurations. Quantities of interest for soil erosion, such as pressure and shear stress at the soil surface have been, therefore, accurately computed, paving the way for quantitative understanding of soil erosion driven by rain. 
The simulations confirm that the maximum shear stress at the soil surface depends in particular on the ratio between the water depth and the drop size. The variation of the pressure inside the water layer during the raindrop impact is well explained by a self-similar approach where the self-similar length is the spreading radius. The position of this radius corresponding to the maximum pressure moves as the square-root of time after impact. Such a relationship comes from very general geometrical arguments and it was in fact previously observed numerically and experimentally for a wider range of drop impacts (especially with drop velocities different from the terminal velocity). Importantly, the present study shows that this relationship is independent of the ratio $h / D$.

At the soil surface, the maximum pressure is located at the center of the impact. Considering half of this pressure, it was found that it moves radially with a square-root of the time after impact only for thin water layers $h / D<1 / 5$. For low $h / D$ ratios, the location of both the maximum pressure inside the water and the pressure at the soil surface follow the same law because the shielding caused by the water layer is minimal. The shielding becomes significant for larger $h / D$ ratios, especially for $h / D \geqslant 1$, leading to a disconnection between pressure behaviors inside the water and at the soil surface. Nevertheless, for all $h / D$ ratios, a self-similarity was found for the pressure rescaled by its central value $P(r, z=0, t)$ as a function of the radius rescaled by the half-pressure radius. The existence of this self-similarity shows that the dynamics of the pressure at the soil surface is quite similar for different $h / D$ ratios (even though the rescaling depends on $h / D$ ).

The shear stress at the soil surface was also rescaled, but the self-similarity was not as consistent as the one for the pressure. This indicates that the dynamics of the shear stress is more complex, and that additional variables may have to be taken into account. In particular, one would need in further study to elucidate the interplay between the growth of the viscous boundary layer and the spreading dynamics.

By clarifying the dynamics of the raindrop impact on a water layer, these results could foster experimental and numerical studies of soil erosion by raindrops. By identifying the variables of interest, it will simplify the design of these studies. More precisely, the equations for the maximum shear stress could be implemented in macroscopic model of erosion to estimate the quantities of materials eroded. New insight could also come from theoretical developments carried out in fluid mechanics, such as the influence of the air cushioning prior to the impact on the interface deformation ( $\mathrm{Xu}$ et al. 2005$)$ or the changes in the flow due to the granular structure of the soil.

Indeed, the biggest drawback of current numerical modeling is probably the hypothesis of a rigid soil surface, which comes from the unavailability of a suitable deformation law. To be realistic such law should account for the aggregated 
status of soils. Finding such a law remains a challenge for soil physicists.

\section{Acknowledgments}

It is our pleasure to thank Leon Malan for his help. C.J. wants to acknowledge the Agence Nationale de la Recherche through the grant ANR "TRAM" 13-BS090011-01.

\section{References}

Afkhami, S., Zaleski, S., and Bussmann, M. (2009). A mesh-dependent model for applying dynamic contact angles to vof simulations. J. Comp. Phys., 228:53705389.

Ahn, S., Doerr, S. H., Douglas, P., Bryant, R., Hamlett, C. A., McHale, G., Newton, M. I., and Shirtcliffe, N. J. (2013). Effects of hydrophobicity on splash erosion of model soil particles by a single water drop impact. Earth Surface Processes and Landforms, 38(11):1225-1233.

Atlas, D., Srivastava, R. C., and Sekhon, R. S. (1973). Doppler radar characteristics of precipitation at vertical incidence. Reviews of Geophysics and Space Physics, 11(1):1-35.

Barrat, J.-L. and Bocquet, L. (1999). Large slip effect at a nonwetting fluid-solid interface. Phys. Rev. Lett., 82:4671.

Brackbill, J., Kothe, D. B., and Zemach, C. (1992). A continuum method for modeling surface tension. J. Comput. Phys., 100:335-354.

Bresson, L. M. and Moran, C. J. (2003). Role of compaction versus aggregate disruption on slumping and shrinking of repacked hardsetting seedbeds. Soil Science, 168(8):585-594.

Charru, F., Mouilleron, H., and Eiff, O. (2004). Erosion and deposition of particles on a bed sheared by a viscous flow. J. Fluid Mech., 519:55-80.

Dunne, T., Malmon, D. V., and Mudd, S. M. (2010). A rain splash transport equation assimilating field and laboratory measurements. Journal of Geophysical Research - Earth Surface, 115:F01001 1-16.

Eggers, J., Fontelos, M., Josserand, C., and Zaleski, S. (2010). Drop dynamics after impact on a solid wall: Theory and simulations. Phys. Fluids, 22:062101 $1-13$. 
Erpul, G., Gabriels, D., and Norton, L. D. (2004). Wind effects on sediment transport by raindrop-impacted shallow flow: A wind-tunnel study. Earth Surface Processes and Landforms, 29:955-967.

Ferreira, A. G., Larock, B. E., and Singer, M. J. (1985). Computer-simulation of water drop impact in a 9.6-mm deep pool. Soil Science Society of America Journal, 49(6):1502-1507.

Ferreira, A. G. and Singer, M. J. (1985). Energy-dissipation for water drop impact into shallow pools. Soil Science Society of America Journal, 49(6):1537-1542.

Ghadiri, H. and Payne, D. (1977). Raindrop impact stress and the breakdown of soil crumbs. Journal of Soil Science, 28:247-258.

Ghadiri, H. and Payne, D. (1980). A study of soil splash using cine-photography. In De Boot, M. and Gabriels, D., editors, Assessment of Erosion, pages 185192. John Wiley \& Sons Ltd.

Ghadiri, H. and Payne, D. (1986). The risk of leaving the soil surface unprotected against falling rain. Soil \& Tillage Research, 8(1-4):119-130.

Hartley, D. M. and Alonso, C. V. (1991). Numerical study of the maximum boundary shear-stress induced by raindrop impact. Water Resources Research, 27(8):1819-1826.

Hartley, D. M. and Julien, P. Y. (1992). Boundary shear-stress induced by raindrop impact. Journal of Hydraulic Research, 30(3):341-359.

Houssais, M. and Lajeunesse, E. (2012). Bedload transport of a bimodal sediment bed. J. Geophys. Res., 117:F04015 1-13.

Huang, C., Bradford, J. M., and Cushman, J. H. (1982). A numerical study of raindrop impact phenomena - the elastic-deformation case. Soil Science Society of America Journal, 47(5):855-861.

Josserand, C. and Thoroddsen, S. (2016). Drop impact on a solid surface. Annu. Rev. Fluid Mech., 48:365-391.

Josserand, C. and Zaleski, S. (2003). Droplet splashing on a thin liquid film. Phys. Fluids, 15:1650-1657.

Kinnell, P. I. A. (1991). The effect of flow depth on sediment transport induced by raindrops impacting shallow flows. Transactions of the ASAE, 34(1):161-168. 
Korobkin, A. A., Ellis, A. S., and Smith, F. T. (2008). Trapping of air in impact between a body and shallow water. J. Fluid Mech., 611:365-394.

Lafaurie, B., Nardone, C., Scardovelli, R., Zaleski, S., and Zanetti, G. (1994). Modelling merging and fragmentation in multiphase flows with SURFER. J. Comput. Phys., 113:134-147.

Lagubeau, G., Fontelos, M., Josserand, C., Maurel, A., Pagneux, V., and Petitjeans, P. (2010). Flower patterns in drop impact on thin liquid films. Phys. Rev. Lett., 105:184503 1-4.

Leguédois, S., Planchon, O., Legout, C., and Le Bissonnais, Y. (2005). Splash projection distance for aggregated soils. theory and experiment. Soil Science Society of America Journal, 69(1):30-37.

Lesser, M. and Field, J. (1983). The impact of compressible liquids. Annu. Rev. Fluid Mech., 15:97.

$\mathrm{Li}$, J. (1995). Calcul d'interface affine par morceaux (piecewise linear interface calculation). C. R. Acad. Sci. Paris, série IIb, (Paris), 320:391-396.

Marengo, M., Antonini, C., Roisman, I. V., and Tropea, C. (2011). Drop collisions with simple and complex surfaces. Current Opinion in Colloid and Interface Science, 16(4):292-302.

Meyer-Peter, E. and Müller, R. (1948). Formulas for bed-load transport. In Proc. 2nd Meet. Int. Assoc. Hydraul. Struct. Res., pages 39-64.

Moss, A. J. and Green, P. (1983). Movement of solids in air and water by raindrop impact. effects of drop-size and water-depth variations. Australian Journal of Soil Research, 21:257-269.

Mouzai, L. and Bouhadef, M. (2011). Shear strength of compacted soil: Effects on splash erosion by single water drops. Earth Surface Processes and Landforms, 36(1):87-96.

Mutchler, C. K. and Young, R. A. (1975). Soil detachment by raindrops. In Present Prospective Technology for Predicting Sediment Yields and Sources, pages 114-117. U.S. Dep. of Agric., Washington, D. C., U.S.A.

Nearing, M. A., Bradford, J. M., and Holtz, R. D. (1986). Measurement of force vs time relations for waterdrop impact. Soil Science Society of America Journal, 50(6):1532-1536. 
Nearing, M. A., Bradford, J. M., and Holtz, R. D. (1987). Measurement of waterdrop impact pressure on soil surfaces. Soil Science Society of America Journal, 51:1302-1306.

Palmer, R. S. (1963). The influence of a thin water layer on waterdrop impact forces. Assoc. Sci. Hydrol. Publ., 65:141-148.

Palmer, R. S. (1965). Waterdrop impact forces. Transactions of the ASAE, 8:69-70.

Parker, G. (1990). Surface-based bedload transport relation for gravel rivers. Journal of Hydraulic Research, 28:417-436.

Planchon, O. and Mouche, E. (2010). A physical model for the action of raindrop erosion on soil microtopography. Soil Sci. Soc. AM. J., 74:1092-1103.

Popinet, S. (2003). Gerris: a tree-based adaptive solver for the incompressible euler equations in complex geometries. J. Comp. Phys., 190(2):572-600.

Popinet, S. (2007). Gerris flow solver. http://gfs.sourceforge.net/

Popinet, S. (2009). An accurate adaptive solver for surface-tension-driven interfacial flows. J. Comput. Phys., 228:5838-5866.

Rein, M. (1993). Phenomena of liquid drop impact on solid and liquid surfaces. Fluid Dyn. Res., 12:61.

Roisman, I. V. (2009). Inertia dominated drop collisions. ii. an analytical solution of the navierstokes equations for a spreading viscous film. Phys. Fluids, 21:052104 1-11.

Sharma, P. P., Gupta, S. C., and Rawls, W. J. (1991). Soil detachment by single raindrops of varying kinetic-energy. Soil Science Society of America Journal, 55(2):301-307.

Singer, M. J., Walker, P. H., Hutka, J., and Green, P. (1981). Soil erosion under simulated rainfall and runoff at varying cover levels. Technical Report 55, Division of Soils, Commonwealth Scientific and Industrial Research Organization, Camberra, Australia.

Terry, J. P. (1998). A rainsplash component analysis to define mechanisms of soil detachment and transportation. Australian Journal of Soil Research, 36(3):525-542. 
Thoroddsen, S. (2002). The ejecta sheet generated by the impact of a drop. Journal of Fluid Mechanics, 451:373.

Thoroddsen, S. T., Etoh, T. G., and Takehara, K. (2003). Air entrapment under an impacting drop. Journal of Fluid Mechanics, 478:125-134.

Thoroddsen, S. T., Etoh, T. G., Takehara, K., Ootsuka, N., and Hatsuki, A. (2005). The air bubble entrapped under a drop impacting on a solid surface. Journal of Fluid Mechanics, 545:203-212.

Torri, D. and Sfalanga, M. (1986). Some aspects of soil erosion modeling. In Giorgini, A. and Zingales, F., editors, Agricultural nonpoint source pollution: Model selection and application, pages 161-171. Elsevier.

Villermaux, E. and Bossa, B. (2009). Single-drop fragmentation determines size distribution of raindrops. Nature Physics, 5:697-702.

Wagner, H. (1932). über stoss und gleitvorgänge und der oberfläshe von flüssigkeiten. Zeit. Angewandte Math. Mech., 12(4):193-215.

Wang, R. C.-T. and Wenzel, Jr, H. G. (1970). The mechanics of a drop after striking a stagnant water layer. Technical Report 30, Water Resources Center, University of Illinois, USA.

Worthington, A. (1876). On the form assumed by drops of liquids falling vertically on a horizontal plate. Proc. R. Soc. Lond., 25:261-271.

Xu, L., Zhang, W., and Nagel, S. (2005). Drop splashing on a dry smooth surface. Phys. Rev. Lett., 94:184505 1-4.

Yarin, A. and Weiss, D. (1995). Impact of drops on solid surfaces: self-similar capillary waves, and splashing as a new type of kinematic discontinuity. Journal of Fluid Mechanics, 283:141-173.

Yarin, A. L. (2006). Drop impact dynamics: Splashing, spreading, receding, bouncing... Annu. Rev. Fluid Mech., 38:159. 